Pacific Journal of Mathematics

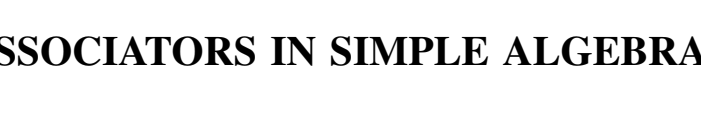




\section{ASSOCIATORS IN SIMPLE ALGEBRAS}

\section{S. ROBERT GORDON}

In this paper it is shown that, with suitable hypotheses on the base field, any element of generic trace zero in an octonion algebra is a commutator and an associator, and any element of generic trace zero in a simple Jordan algebra is an associator.

In $1937 \mathrm{~K}$. Shoda [7] proved that every $n \times n$ matrix $A$ of trace zero over a field of characteristic bigger than $n$ is a commutator $[B, C]=B C-C B$ for suitable $n \times n$ matrices $B, C$. His method was to show that $A$ is similar to a matrix all of whose diagonal entries are zero, and then to give a specific formula for such a matrix. In 1957 A. A. Albert and B. Muckenhoupt [1] proved this theorem for arbitrary fields by proving a more complicated similarity theorem and giving a more complicated formula. In $1963 \mathrm{G}$. Brown [3] proved an analogous theorem for Lie algebras: that every element of a (split) classical Lie algebra $\&$ is a commutator. His result is valid over all fields, with the exception of certain small finite fields. His method was to show that if $\mathfrak{L}=\mathfrak{S} \oplus \sum_{\alpha \neq 0} \mathfrak{L}_{\alpha}$ is the Cartan decomposition of $\mathfrak{Z}$ with respect to a Cartan subalgebra $\mathfrak{K}$, then every element of $\mathbb{R}$ is conjugate under the automorphism group of $\mathbb{R}$ to an element of $\sum_{\alpha \neq 0} \mathbb{R}_{\alpha}$.

In this paper we present similar results for alternative and Jordan algebras. If $\mathfrak{Y}$ is a (nonassociative) algebra with multiplication $x, y \mapsto x y$, we define the commutator of $x, y \in \mathfrak{N}$ to be $[x, y]=x y-y x$ and the associator of $x, y, z \in \mathfrak{N}$ to be $[x, y, z]=(x y) z-x(y z)$. We prove that in an octonion algebra (with the possible exception of division algebras of characteristic 2) any element of trace zero is both a commutator and an associator. We show that in a simple Jordan algebra over an algebraically closed field of characteristic bigger than the degree of the algebra, every element of trace zero is an associator (the question of commutators does not arise). Our methods are analogous to the above: in each case we prove that an element of trace zero is conjugate under the automorphism group of the algebra to one whose "diagonal entries" (in an appropriate sense) are zero. Then we give a specific computation for such elements.

The product of two elements $x, y$ of an associative or octonion algebra will be denoted $x y$; the product of elements $x, y$ of a Jordan algebra will be denoted $x, y$. The reader is referred to [2] and [6] for relevant properties of octonion algebras, and to [5], especially Chapter 5 , §6, for properties of simple Jordan algebras. 
1. Octonion algebras. We prove the following:

THEOREM 1. Let $\mathfrak{O}$ be either an octonion division algebra over a field $\Phi$ of characteristic $\neq 2$ or a split octonion algebra over an arbitrary field $\Phi$. Then every element of $\mathfrak{O}$ of trace zero is both a commutator $[a, b]$ and an associator $[a, b, c]$.

Proof. We denote the canonical involution on $\subseteq$ by $x \mapsto \bar{x}$. The generic trace and norm forms on $\mathfrak{D}$ are $x+\bar{x}=t(x), x \bar{x}=\bar{x} x=n(x)$ respectively. The linearized norm form is $n(x, y)=x \bar{y}+y \bar{x}=t(x \bar{y})$. See [2] or [6] for properties of these forms.

If $\mathfrak{O}$ is a division algebra, we give an argument of $R$. Brown. Suppose $a \in \mathfrak{O}$ and $t(a)=0$. Choose $0 \neq b \in \mathfrak{O}$ such that $n(1, b)=$ $n(a, b)=0$; this is possible since $\langle 1, b\rangle$ must be at least 6 -dimensional, hence nonzero. Since $n(1, b)=t(b)$, we have $\bar{a}=-a, \bar{b}=-b, 0=$ $a \bar{b}+b \bar{a}=-a b-b a$. Using the alternative law, we have

$$
\begin{aligned}
{[b, a b] } & =b(a b)-(a b) b=-b(b a)-(a b) b=-b^{2} a-a b^{2} \\
& =(b \bar{b}) a+a(b \bar{b})=2 n(b) a .
\end{aligned}
$$

Since $2 \neq 0$ and $n(b) \neq 0$ ( $\subseteq$ is a division algebra), $a$ is a commutator.

Now choose $0 \neq c \in \mathfrak{D}$ orthogonal to $1, a, b, a b$ : the orthogonal compliment of these elements is at least 4-dimensional. Then

$$
\begin{aligned}
(a b) c-a(b c) & =[a, b, c]=[c, a, b]=(c a) b-c(a b) \\
& =(c a) b-c(\bar{a} \bar{b})=(c a) b-c(\overline{b a})=(c a) b+c(\overline{a b}) \\
& =(c a) b-(a b) \bar{c}=(c a) b+(a b) c \\
a(b c) & =-(c a) b \\
{[a(b c), b, c] } & =((a(b c)) b) c-(a(b c))(b c) \\
& =(((c a) b) b) c-a(b c)^{2} .
\end{aligned}
$$

Since $n(b, c)=0$ implies $\overline{b c}=-b c$ and $n(a, c)=0$ implies $c a=-a c$, we have

$$
\begin{aligned}
{[a(b c), b, c] } & =\left((c a) b^{2}\right) c+a n(b c)=n(b)(c a c)+a n(b c) \\
& =-n(b)\left(a c^{2}\right)+a n(b c)=n(b) n(c) a+a n(b c) \\
& =2 n(b) n(c) a .
\end{aligned}
$$

But $2, n(b), n(c)$ are all nonzero, so $a$ is an associator.

We now assume that $\mathfrak{D}$ is a split octonion algebra. Let $x_{0}, y_{0}, x_{1}$, $y_{1}, x_{2}, y_{2}, x_{3}, y_{3}$ be a canonical basis for $\mathfrak{O}$ as given by van der Blij and Springer [2, p. 410-411]; $\left\{x_{2}, y_{i}\right\}$ are mutually orthogonal hyperbolic pairs and $x_{0}, y_{0}$ are complimentary primitive idempotents. Suppose $v \in \mathfrak{D}$ and $t(v)=0$. Suppose furthermore that $1, v$ are linearly inde- 
pendent (this happens necessarily if the characteristic is not 2). Let $n(v)=\alpha \in \Phi$. Then $n\left(x_{1}+\alpha y_{1}\right)=n\left(x_{1}\right)+n\left(x_{1}, \alpha y_{1}\right)+n\left(\alpha y_{1}\right)=0+\alpha \cdot 1+0=$ $n(v), n\left(1, x_{1}+\alpha y_{1}\right)=n\left(x_{0}+y_{0}, x_{1}+\alpha y_{1}\right)=0=n(1, v)$. Witt's theorem therefore implies that there is an orthogonal transformation $\theta$ of $\mathfrak{D}$ sending $1 \rightarrow 1$ and $x_{1}+\alpha y_{1} \rightarrow v$. Let $e=\theta\left(x_{0}\right), f=1-e=\theta\left(y_{0}\right)$. Then $n(e)=n\left(\theta x_{0}\right)=n\left(x_{0}\right)=0, t(e)=n(e, 1)=n\left(\theta x_{0}, \theta 1\right)=n\left(x_{0}, 1\right)=t\left(x_{0}\right)=1$. Hence $e$ is a primitive idempotent of $\mathfrak{D}$. Also

$$
\begin{gathered}
n(e, v)=n\left(\theta x_{0}, \theta\left(x_{1}+\alpha y_{1}\right)\right)=n\left(x_{0}, x_{1}+\alpha y_{1}\right)=0 \\
n(f, v)=n\left(y_{0}, x_{1}+\alpha y_{1}\right)=0 .
\end{gathered}
$$

Using the arguments of $[2$, p. 411], we can identify $\mathfrak{S}$ with the algebra of "vector matrices" over $\Phi$ in such a way that $e, f$ are identified with the diagonal idempotents. Then $v \in\langle e, f\rangle^{\perp}$ is a matrix with only zeroes on the diagonal. This proves the "conjugacy" step.

We note the following formulas in the algebra of vector matrices:

$$
\begin{aligned}
{\left[\left(\begin{array}{rr}
0 & -a \\
b & 0
\end{array}\right),\left(\begin{array}{ll}
1 & 0 \\
0 & 0
\end{array}\right)\right] } & =\left(\begin{array}{ll}
0 & a \\
b & 0
\end{array}\right) \\
{\left[\left(\begin{array}{ll}
0 & a \\
b & 0
\end{array}\right),\left(\begin{array}{ll}
0 & c \\
d & 0
\end{array}\right),\left(\begin{array}{ll}
1 & 0 \\
0 & 0
\end{array}\right)\right] } & =\left(\begin{array}{cc}
0 & -b \times d \\
a \times c & 0
\end{array}\right)
\end{aligned}
$$

where as usual $a \times b$ is the usual vector product in $\Phi^{3}$, three-dimensional space over $\Phi$. This proves that $v$ is a commutator; to prove that $v$ is an associator one need only show that every element of $\Phi^{3}$ is of the form $a \times b$ for some $a, b \in \Phi^{3}$. Fix $0 \neq a \in \Phi^{3}$. By $a d a$ we mean the map $b \mapsto a \times b$. Since $a \cdot(a \times b)=0$ (here $a \cdot b$ is the usual scalar product on $\left.\Phi^{3}\right)$, the image of ad $a$ is contained in the kernel of the linear functional $b \mapsto a \cdot b$. This kernel is 2-dimensional. If $a=(\alpha, \beta, \gamma)$ then the matrix of ad $a$ is

$$
\left(\begin{array}{rrr}
0 & -\gamma & \beta \\
\gamma & 0 & -\alpha \\
-\beta & \alpha & 0
\end{array}\right)
$$

hence has rank 2. This means that the image of ad a equals the kernel of $b \mapsto a \cdot b$. Given $b \in \Phi^{3}$, choose $0 \neq a \in \Phi^{3}$ so that $a \cdot b=0$. Then $b \in$ Image $(a d a)$, i.e., $b=a \times c$ for some $c$.

We have assumed that $1, v$ are linearly independent. Finally, we need to show that $1 \in \mathfrak{D}$ is a commutator and an associator in characteristic 2. If $\left\{x_{i}, y_{i}\right\}$ is a canonical basis of $\mathfrak{D}$ as above then the multiplication table given in [2] yields

$$
\begin{aligned}
& {\left[x_{1}+y_{1}, x_{1}\right]=x_{0}-y_{0}} \\
& {\left[x_{1}, x_{2}, x_{3}\right]=x_{0}-y_{0} .}
\end{aligned}
$$


This proves the theorem.

It would of course be interesting to know whether these results hold in division algebras of characteristic $2 .^{1}$

2. Jordan algebras. In this section we prove the following:

THEOREM 2. Let $\mathfrak{\Im}$ be a simple Jordan algebra over an algebraically closed field $\Phi$ of characteristic bigger than the degree of $\mathfrak{\Im}$. Then every element of $\mathfrak{\Im}$ of generic trace 0 is an associator $[a, b, c]$.

The assumption of algebraic closure is used to simplify appeal to the classification theory and to guarantee the existence of square roots in the field. It will be seen that at least one crucial lemma would be false otherwise. The theorem may hold more generally, but a different proof would be required. In any event, henceforth $\Phi$ is algebraically closed.

We first note that the theorem is vacuous for algebras of degree 1 (i.e., for $\Phi$ ) and easy for algebras of degree 2. The latter are the Jordan algebras $\Phi 1 \oplus \mathfrak{B}, \mathfrak{B}$ a vector space (of dimensional at least 2) with a nondegenerate symmetric bilinear form (,). $\mathfrak{S}$ is the space of elements of trace zero in this algebra. If $v \in \mathfrak{B}$, choose $0 \neq u \in \mathfrak{S}$ such that $(u, v)=0$ and $w \in \mathfrak{B}$ such that $(u, w)=1$. Then $[w, u, v]=v$.

In proving Theorem 2 for algebras of degree at least 3 , we will consider the algebras $\mathfrak{S}_{\mathfrak{c}}\left(\Phi_{n}\right)$ of symmetric $n \times n$ matrices over $\Phi$ separately from the other algebras.

LEMma 1. Let $\mathfrak{I}=\Phi 1 \oplus \mathfrak{B}$ be the Jordan algebra of the space $\mathfrak{B}$ equipped with the nondegenerate symmetric bilinear form (, ); let $e$ be a primitive idempotent in $\mathfrak{\Im}$ and $f=1-e$. Suppose $a=\alpha e+$ $\beta f+x$ (where $\alpha, \beta \in \Phi, x \in \widetilde{\Im}_{1 / 2}(e)$ ) and suppose $a \in \Phi 1$, $\operatorname{dim} \mathfrak{B} \geqq 3$.

Then there is an automorphism of $\mathfrak{\Im}$ having determinant 1 and sending $a \rightarrow(\alpha+\beta) e+y$, for some $y \in \mathfrak{\Im}_{1 / 2}(e)$.

Proof. Let $v=e-f \in$ S. Then $e=\frac{1}{2}(1+v), f=\frac{1}{2}(1-v)$, $(v, v)=v^{2}=1, \mathfrak{J}_{1 / 2}(e)=\langle v\rangle^{\perp}=\{w \in \mathfrak{B} \mid(v, w)=0\}$. If $y \in \mathfrak{I}_{1 / 2}(e)$ then

$$
\begin{gathered}
\alpha e+\beta f+x=\frac{1}{2}(\alpha+\beta) 1+\frac{1}{2}(\alpha-\beta) v+x \\
(\alpha+\beta) e+y=\frac{1}{2}(\alpha+\beta) 1+\frac{1}{2}(\alpha+\beta) v+y .
\end{gathered}
$$

Since $a$ is not a scalar, either $\alpha \neq \beta$ or $x \neq 0$; in any event, $\frac{1}{2}(\alpha-\beta) v+$

${ }^{1}$ Since the above was written, Michel Racine has supplied the author with a proof of Theorem 1 in this case. 
$x \neq 0$. It therefore suffices to find $y \in \Im_{1 / 2}(e)$ so that $\frac{1}{2}(\alpha-\beta) v+x$ is nonzero and has the same norm as $\frac{1}{2}(\alpha+\beta) v+y$. For then Witt's theorem implies that there is an automorphism $\phi$ of $\mathfrak{I}$ sending the first of the above elements to the second (recall that the automorphism group of $\mathfrak{J}$ is naturally isomorphic to the orthogonal group of $\mathfrak{B}$ ). Since $\operatorname{dim} \mathfrak{B} \geqq 3,\left\langle\frac{1}{2}(\alpha+\beta) v+y\right\rangle^{\perp}$ cannot be a totally isotropic subspace; if $w$ is a nonsingular element of it and $\sigma_{w}$ is the reflection along $w$, then $\operatorname{det}\left(\sigma_{w} \phi\right)=-\operatorname{det} \phi$. The desired automorphism is either $\phi$ or $\sigma_{w} \phi$ : whichever has determinant 1 .

The norm of $\frac{1}{2}(\alpha-\beta) v+x$ is $\left(\frac{1}{2}(\alpha-\beta)\right)^{2}+(x, x)=\frac{1}{4}\left(\alpha^{2}+\right.$ $\left.\beta^{2}\right)-\frac{1}{2} \alpha \beta+(x, x)$. The norm of $\frac{1}{2}(\alpha+\beta) v+y$ is $\frac{1}{4}\left(\alpha^{2}+\beta^{2}\right)+$ $\frac{1}{2} \alpha \beta+(y, y)$. So we need to find $y \in\langle v\rangle$ such that the norm of $y$ is $(y, y)=(x, x)-\alpha \beta$. Since $\langle v\rangle^{\perp}$ is a nonisotropic subspace of dimension at least 2 , and since $\Phi$ is algebraically closed, $\langle v\rangle^{\perp}$ contains nonzero elements of arbitrary norm (including 0 ). So $y$ can be chosen to be nonzero, and hence $\frac{1}{2}(\alpha+\beta) v+y \neq 0$.

Lemma 2. Let $\mathfrak{\Im}$ be a simple Jordan algebra over $\Phi$ and let $e_{1}, \cdots, e_{n}$ be a complete set of orthogonal primitive idempotents in $\Im$. Let $\mathfrak{\Im}=\sum_{i \leqq j} \mathfrak{\Im}_{i j}$ be the corresponding Peirce decomposition and suppose $\operatorname{dim} \Im_{12} \geqq 2$. If $a \in \Im$, say $a=\sum_{i} \alpha_{i} e_{i}+\sum_{i<j} x_{i j}\left(\alpha_{i} \in \Phi, x_{i j} \in \Im_{i j}\right)$, and if either $\alpha_{1} \neq \alpha_{2}$ or $x_{12} \neq 0$, then there is an automorphism of $\Im$ sending $a$ to an element $\left(\alpha_{1}+\alpha_{2}\right) e_{2}+\sum_{i=3}^{n} \alpha_{i} e_{i}+\sum_{i<j} y_{i j}$ for some $y_{i j} \in \Im_{i j}$.

Proof. We apply Lemma 1 to the simple Jordan algebra $\mathfrak{A}=\mathfrak{J}_{11} \oplus$ $\mathfrak{I}_{12} \oplus \mathfrak{\Im}_{22}$, and conclude that there is an automorphism $\phi$ of this algebra, of determinant 1 and sending $\alpha_{1} e_{1}+\alpha_{2} e_{2}+x_{12}$ to $\left(\alpha_{1}+\alpha_{2}\right) e_{2}+y_{12}$ for

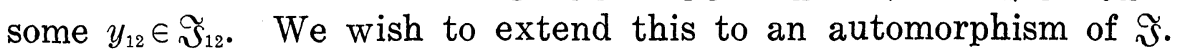

We note that in a simple Jordan algebra $\Phi 1 \oplus \mathfrak{B}$ any automorphism of determinant 1 is a product of maps $U_{2 e-1} U_{2 f-1}$ ( $U$ the quadratic Jordan operator, $e$ and $f$ primitive idempotents). For if $v=2 e-1 \in \mathfrak{B}$, then $U_{2 e-1}(1)=2(1 . v) . v-1 . v^{2}=2-1=1$; and $w \in \mathfrak{B}$ implies

$$
U_{2 e-1}(w)=2(v, w) v-w(v, v)=-\left(w-\frac{2(v, w)}{(v, v)} v\right) .
$$

I.e., $U_{2 e-1}=-\sigma_{v}$ (where $\sigma_{v}$ is the reflection along $v$ ). But every orthogonal map of $\mathfrak{B}$ of determinant 1 is the product of an even 
number of reflections, hence of an even number of negatives of reflections; and any reflection is a reflection along a vector of norm 1 , since the field is algebraically closed.

We conclude that $\phi$ is a product of automorphism $U_{2 e-\left(e_{1}+e_{2}\right)}$, where $e$ is a primitive idempotent of $\mathfrak{A}$. But $U_{2 e-1}$ restricted to $\mathfrak{A}$ is $U_{2 e-\left(e_{1}+e_{2}\right)}$, since $U_{2 e-1}(x)=8(x \cdot e) \cdot e-8 x \cdot e+x$ for all $x \in \mathfrak{\Im}$ and $U_{2 e-\left(e_{1}+e_{2}\right)}(x)$ is the same for $x \in \mathfrak{A}[4, \mathrm{p}$. 47]. Thus $\phi$ extends to an automorphism $\phi$ of $\mathfrak{\Im}$ which is a product of automorphisms the form $U_{2 e-1}, e$ a primitive idempotent of $\mathfrak{A}$. We show this $\phi$ has the desired property.

Let $e$ be a primitive idempotent of $\mathfrak{A}, f=e_{1}+e_{2}-e . \quad U_{2 e-1}$ is the identity on $\mathfrak{\Im}_{0}(e) \oplus \mathfrak{\Im}_{1}(e)$ and is -1 on $\mathfrak{\Im}_{1 / 2}(e)$. We have the Peirce decomposition $\mathfrak{\Im}=\sum_{i \leqq j} \mathfrak{\Im}_{i j}^{\prime}$ with respect to the idempotents $e, f, e_{3}$, $\cdots, e_{n}$. So $U_{2 e-1}$ is the identity on $\sum_{\imath, j \geqq 3} \mathfrak{\Im}_{i j}$. If $i \geqq 3$ then $U_{2 e-1}$ is -1 on $\mathfrak{\Im}_{1 i}^{\prime}$ and +1 on $\mathfrak{\Im}_{2 i}^{\prime}$, hence stabilizes $\mathfrak{\Im}_{1 i}^{\prime} \oplus \mathfrak{\Im}_{2 i}^{\prime}=\mathfrak{\Im}_{1 / 2}(e+f) \cap$ $\Im_{1 / 2}\left(e_{i}\right)=\Im_{1 / 2}\left(e_{1}+e_{2}\right) \cap \Im_{1 / 2}(e)=\Im_{1 i} \bigoplus \Im_{2 i}$. We conclude that $\phi$ is the identity on $\mathfrak{\Im}_{i j}(i, j \geqq 3)$ and stabilizes all $\Im_{1 i} \oplus \Im_{2:}(i \geqq 3)$; hence $\phi$ has the desired effect on $a$.

The next lemma is the conjugacy step in the argument. We recall that in a simple Jordan algebra over $\Phi$ of degree at least 3 all the Peirce spaces $\Im_{i j}(i \neq j)$ have the same dimension $1,2,4$ or 8 [5].

LeMma 3. Let $\Im$ be a simple Jordan algebra over $\Phi$ and $e_{1}, \cdots, e_{n}$ a complete set of orthogonal primitive idempotents. Assume the Peirce spaces $\Im_{i j}(i \neq j)$ with respect to these idempotents are not one-dimensional. Assume also that the characteristic of $\Phi$ is 0 or bigger than $n$. Then every element of $\mathfrak{\Im}$ of trace 0 is conjugate under the automorphism group of $\mathfrak{\Im}$ to an element of $\sum_{i<j} \mathfrak{\Im}_{i j}$.

Proof. Let $a=\sum_{i} \alpha_{i} e_{i}+\sum_{i<j} x_{i j}$ with $\sum_{i} \alpha_{i}=0$. We show by induction on $k$ that $a$ is conjugate to an element $\sum \beta_{i} e_{i}+\sum y_{i j}$ in which at least $k$ of the $\beta_{i}$ 's are zero. If $k=0$, there is nothing to prove. Assume the result for $k$. The nonzero $\beta_{i}$ 's cannot all be equal, say, to $\beta$; otherwise $(n-k) \beta=\operatorname{tr}(\alpha)=0$ : but $n-k \neq 0$ by the assumption on the characteristic. So choose $i, j$ such that $\beta_{\imath} \neq \beta_{j}$ are nonzero. We apply Lemma 2 and conclude that $a$ is conjugate to an element with $k+1$ zeroes among the coefficients of the idempotents.

We note that this conjugacy theorem is false if $\operatorname{dim} \mathfrak{\Im}_{j}=1$. Let $\mathfrak{\Im}=\mathfrak{S}_{\mathfrak{C}}\left(\Phi_{n}\right)$ be the algebra of $n \times n$ symmetric matrices over $\Phi$. Let $A$ be the matrix with $\left(\begin{array}{rr}1 & i \\ i & -1\end{array}\right)$ in the upper left-hand corner and zero elsewhere (here $i=\sqrt{-1}$ ). Then $A$ is not orthogonally similar to any symmetric matrix all of whose diagonal entries are zero. To see this, 
we regard $\mathfrak{\Im}$ as acting on the vector space $\mathfrak{B}$ with the nondegenerate symmetric bilinear form (,) and orthogonal basis $x_{1}, \cdots, x_{n}$. Let $A$ be the linear transformation of $\mathfrak{B}$ whose matrix with respect to this basis is $A$. Suppose $y_{1}, \cdots, y_{n}$ is another orthonormal basis with respect to which the matrix of $A$ has all zeroes on the diagonal. Let $y_{k}=\sum_{j} \alpha_{j k} x_{j}$. Then

$$
\begin{aligned}
0 & =\left(A y_{k}, y_{k}\right) \\
& =\left(\alpha_{1 k}\left(x_{1}+i x_{2}\right)+\alpha_{2 k}\left(i x_{1}-x_{2}\right), \alpha_{1 k} x_{1}+\alpha_{2 k} x_{2}+\cdots+\alpha_{n k} x_{n}\right) \\
& =\alpha_{1 k}^{2}+2 i \alpha_{1 k} \alpha_{2 k}-\alpha_{2 k}^{2}=\left(\alpha_{1 k}+i \alpha_{2 k}\right)^{2} .
\end{aligned}
$$

Hence for all $k, \alpha_{1 k}+i \alpha_{2 k}=0$. This says that all $y_{k}$ belong to some $(n-1)$-dimensional subspace of $\mathfrak{B}$, a contradiction.

Finally, we prove Theorem 2 for Jordan algebras $\mathfrak{I}$ in which the Peirce spaces $\mathfrak{\Im}_{i j}$ are not one-dimensional. The notation is as in Lemmas 2 and 3 . If $a=\sum_{i<j} x_{i j}$, we show $a$ is an associator. By Lemma 3 , this is sufficient. But

$$
\begin{aligned}
& {\left[y_{i j}, \alpha_{i} e_{i}+\alpha_{j} e_{j}, \alpha_{\imath} e_{\imath}+\alpha_{\jmath} e_{j}\right]} \\
& \quad=\frac{1}{4}\left(\alpha_{\imath}+\alpha_{j}\right)^{2} y_{\imath j}-y_{\imath j} \cdot\left(\alpha_{i}^{2} e_{\imath}+\alpha_{j}^{2} e_{j}\right) \\
& \quad=\frac{1}{4}\left(\alpha_{\imath}+\alpha_{j}\right)^{2} y_{i j}-\frac{1}{2}\left(a_{i}^{2}+\alpha_{j}^{2}\right) y_{i j}=-\frac{1}{4}\left(\alpha_{\imath}-\alpha_{j}\right)^{2} y_{\imath j},
\end{aligned}
$$

if $y_{\imath, j} \in \mathfrak{\Im}_{i j}$. So

$$
\left[\sum_{i<j} y_{i j}, \sum_{i} \alpha_{2} e_{i}, \sum_{i} \alpha_{\imath} e_{i}\right]=\sum_{i<j}-\frac{\left(\alpha_{2}-\alpha_{j}\right)^{2}}{4} y_{i j} .
$$

If we take $\alpha_{1}, \cdots, \alpha_{n}$ to be distinct and $y_{\imath j}=-4\left(\alpha_{\imath}-\alpha_{j}\right)^{-2} x_{\imath j}$, we see that $a$ is an associator.

We now prove Theorem 2 under the assumption that $\mathfrak{\Im} \cong \mathscr{S}\left(\Phi_{n}\right)$ for some $n \geqq 3$; by the classification theory of Jordan algebras, this is the only remaining case. We regard $\mathfrak{\Im}$ as the algebra of symmetric operators on the space $\mathfrak{B}$ as above. We will have occasion to use both orthonormal and hyperbolic bases of $\mathfrak{B}$; the latter are bases $x_{1}, \cdots, x_{l}, y_{1}, \cdots, y_{l}$ or $x_{1}, \cdots, x_{l}, y_{1}, \cdots, y_{l}, z$ (depending on whether $\operatorname{dim} \mathfrak{B}$ is even or odd) satisfying $\left(x_{i}, x_{j}\right)=\left(y_{i}, y_{j}\right)=\left(x_{i}, z\right)=\left(y_{i}, z\right)=0$, $\left(x_{\imath}, y_{j}\right)=\delta_{\imath j},(z, z)=1$. The existence of bases of both kinds follows from the algebraic closure of the field.

LeMma 4. Let $\mathfrak{B}$ be a three-dimensional vector space over $\Phi$ with nondegenerate symmetric bilinear form (,). Let $\sigma$ be a nonscalar symmetric linear transformation on $\mathfrak{B}$. Then there exists a hyperbolic basis $x, y, z$ of $\mathfrak{B}$ with respect to which the matrix of $\sigma$ has the form 


$$
\left(\begin{array}{lll}
0 & \beta & \nu \\
\gamma & 0 & \mu \\
\mu & \nu & \lambda
\end{array}\right)
$$

Proof. Since the matrix of a symmetric transformation with respect to a hyperbolic basis has the form

$$
\left(\begin{array}{lll}
\alpha & \beta & \nu \\
\gamma & \alpha & \mu \\
\mu & \nu & \lambda
\end{array}\right)
$$

we need to find a hyperbolic basis $x, y, z$ such that $\sigma x$ is a linear combination of $y$ and $z$. We first note that there exists $0 \neq x \in \mathfrak{B}$ such that $(x, x)=0$ and $x, \sigma x$ are linearly independent. Suppose otherwise. Let $x, y, z$ be a hyperbolic basis of $\mathfrak{B}$. Then $(x-(1 / 2) y+$ $z, x-(1 / 2) y+z)=2(-1 / 2)+1=0,(x, x)=0,(y, y)=0$. So if the matrix of $\sigma$ is as above, then $\sigma x=\alpha x, \sigma y=\alpha y, \gamma=\mu=\beta=\nu=0$, and hence $\sigma z=\lambda z$. Also $\sigma(x-(1 / 2) y+z)=\alpha x-(1 / 2) \alpha y+\lambda z$ is a scalar multiple of $x-(1 / 2) y+z$; this implies that $\lambda=\alpha$, hence that $\sigma=\alpha 1$ is a scalar, a contradiction.

Suppose then that $(x, x)=0$ and $x, \sigma x$ are linearly independent. Suppose in addition that $(\sigma x, x)=0$. There exists $y^{\prime} \in \mathfrak{B}$ such that $\left(x, y^{\prime}\right)=1,\left(\sigma x, y^{\prime}\right)=0$. The two-dimensional subspace $\left\langle x, y^{\prime}\right\rangle$ is nonisotropic since the matrix of the form on it is nonsingular. So there exists $y^{\prime \prime} \in\left\langle x, y^{\prime}\right\rangle$ such that $\left\langle x, y^{\prime \prime}\right\rangle=1,\left\langle y^{\prime \prime}, y^{\prime \prime}\right\rangle=0$. Since $0 \neq \sigma x \in$ $\left\langle x, y^{\prime}\right\rangle^{\perp}=\left\langle x, y^{\prime \prime}\right\rangle^{\perp}, \sigma x$ must be a nonsingular vector (otherwise it would be orthogonal to $x, y^{\prime \prime}, \sigma x$, hence to all of $\left.\mathfrak{B}\right)$. So $x, y^{\prime \prime}, z=x / \sqrt{(\sigma x, \sigma x)}$ is a hyperbolic basis of $\mathfrak{B}$ of the desired kind.

Finally, suppose $(\sigma x, x) \neq 0$. Embed $x$ in the hyperbolic basis $x, y, z$ and let $\sigma x=\alpha x+\gamma y+\mu z$. Note that $(\sigma x, x)=\gamma \neq 0$. Let

$$
\mu_{1}=\mu+\sqrt{(\sigma x, \sigma x)}=\mu+\sqrt{\mu^{2}+2 \alpha \gamma} .
$$

Hence $\left(\mu_{1}-\mu\right)^{2}=\mu^{2}+2 \alpha \gamma$ and thus $\mu_{1}^{2}-2 \mu \mu_{1}=2 \alpha \gamma$. Let

$$
\begin{aligned}
& \alpha_{1}=-\mu_{1}^{2} / 2 \gamma=-\left(2 \alpha \gamma+2 \mu \mu_{1}\right) / 2 \gamma=-\alpha-\mu \mu_{1} / \gamma \\
& y_{1}=\alpha_{1} x+\gamma y+\mu_{1} z \\
& z_{1}=\sigma x-y_{1} .
\end{aligned}
$$

Then

$$
\begin{aligned}
& \left(y_{1}, \sigma x\right)=\alpha_{1} \gamma+\gamma \alpha+\mu \mu_{1}=\left(-\alpha-\mu \mu_{1} / \gamma\right) \gamma+\gamma \alpha+\mu \mu_{1}=0 \\
& \left(y_{1}, y_{1}\right)=2 \alpha_{1} \gamma+\mu_{1}^{2}=2\left(-\mu_{1}^{2} / 2 \gamma\right) \gamma+\mu_{1}^{2}=0
\end{aligned}
$$




$$
\begin{aligned}
\left(z_{1}, x\right) & =(\sigma x, x)-\left(y_{1}, x\right)=\beta-\beta=0 \\
\left(z_{1}, y_{1}\right) & =\left(\sigma x-y_{1}, y_{1}\right)=0 .
\end{aligned}
$$

Since $(x, x)=0$ and $\left(x, y_{1}\right)=\gamma$, we see that $x, y_{1} / \gamma, z_{1} / \sqrt{\left(z_{1}, z_{1}\right)}$ is a hyperbolic basis of $\mathfrak{B}$. It is as desired since $\sigma x=y_{1}+z_{1}$. This proves the lemma.

Note that the lemma is false without the assumption that the field is algebraically closed. Suppose $\mathfrak{B}$ is a real vector space with hyperbolic basis $x, y, z$. Let $\sigma$ be the linear transformation whose matrix with respect to this basis is

$$
\left(\begin{array}{rrr}
1 & -3 & 0 \\
-3 & 1 & 0 \\
0 & 0 & -2
\end{array}\right)
$$

Let $x^{\prime}=\theta x+i y+\phi z$ be a singular vector: i.e., $2 \theta \psi+\phi^{2}=0$. Then $\sigma\left(x^{\prime}\right)=(\theta-3 \psi) x+\left(-3 \theta+\psi^{\prime}\right) y-2 \phi z$, so

$$
\begin{aligned}
\left(\sigma x^{\prime}, \sigma x^{\prime}\right) & =2(\theta-3 \psi)(-3 \theta+\psi)+4 \phi^{2} \\
& =2\left(-3 \theta^{2}+10 \psi \theta-3 \psi^{2}-4 \theta \psi\right) \\
& =-2\left(3 \theta^{2}-6 \psi \theta+3 \psi^{2}\right)=-6(\theta-\psi)^{2} \leqq 0 .
\end{aligned}
$$

Suppose $x^{\prime}, y^{\prime}, z^{\prime}$ is a hyperbolic basis of $\mathfrak{B}$ with respect to which the matrix of $\sigma$ is as required in Lemma 4. Then $\left(\sigma x^{\prime}, \sigma x^{\prime}\right)=\left(\gamma y^{\prime}+\right.$ $\left.\mu z^{\prime}, \gamma y^{\prime}+\mu z^{\prime}\right)=\mu^{2} \geqq 0$. We conclude that $\mu=0$. Also $\left(\sigma y^{\prime}, \sigma y^{\prime}\right)=$ $\nu^{2} \geqq 0$ so $\nu=0$. And $\lambda=0$ since $\lambda$ is the trace of $\sigma$. Therefore, the matrix of $\sigma$ with respect to $x^{\prime}, y^{\prime}, z^{\prime}$ is

$$
\left(\begin{array}{lll}
0 & \beta & 0 \\
\gamma & 0 & 0 \\
0 & 0 & 0
\end{array}\right),
$$

which is impossible since $\sigma$ is nonsingular.

The next lemma is the conjugacy step.

Lemma 5 . Let $\mathfrak{B}$ be an $n$-dimensional vector space over $\Phi(n \geqq 2)$ with nondegenerate symmetric bilinear form (, ). Let $\sigma$ be a symmetric linear transformation on $\mathfrak{B}$ of trace zero. Assume the characteristic of $\Phi$ is 0 or bigger than $n$. Then there is a hyperbolic basis of $\mathfrak{B}$ with respect to which the matrix of $\sigma$ has all diagonal entries equal to zero.

Proof. If $\mathfrak{U}$ is a nonisotropic subspace of $\mathfrak{B}$ then $\mathfrak{B}=\mathfrak{U} \oplus \mathfrak{U}{ }^{\perp}$. For $x \in \mathfrak{U}$ define $\sigma_{\mathfrak{u}}(x)$ to be that element $u \in \mathfrak{U}$ such that there exists $v \in U$ with $\sigma x=u+v$. Then clearly $\sigma_{\mathfrak{u}}: \mathfrak{u} \rightarrow \mathfrak{u}$ is a symmetric linear 
transformation on $\mathfrak{U}$. The matrix of $\sigma$ with respect to a basis of $\mathfrak{B}$ obtained by combining bases of $\mathfrak{U}$ and $\mathfrak{u}$ has the form $\left(\begin{array}{ll}A & B \\ C & D\end{array}\right)$, where $A$ is the matrix of $\sigma_{\mathfrak{n}}$ and $D$ the matrix $\sigma_{\mathfrak{u}}$.

We proceed by induction on $n$. The lemma is trivially true if $n=2$ (in this case any hyperbolic basis will do, since the diagonal entries in the matrix of $\sigma$ must be equal, hence both zero) and true if $n=3$ by Lemma 4 (note that $\sigma$ is not a scalar, since $3 \neq 0$ ). Assume $n \geqq 4$. It will be sufficient to find a 2 -dimensional nonisotropic subspace $\mathfrak{U}$ of $\mathfrak{B}$ such that $\operatorname{tr}\left(\sigma_{\mathfrak{u}}\right)=0$. For by induction the lemma applies to $\sigma_{\mathfrak{u}}\left\llcorner\right.$, which has dimension $n-2 \geqq 2$; note that $\operatorname{tr}\left(\sigma_{\mathfrak{u}\lrcorner}\right)=\operatorname{tr}(\sigma)-$ $\operatorname{tr}\left(\sigma_{\mathfrak{u}}\right)=0$. So if $x_{1}, y_{1}$ is any hyperbolic basis of $\mathfrak{U}$ and $x_{2}, y_{2}, \cdots$ is the hyperbolic basis of $\mathfrak{u}$ whose existence is guaranteed by induction, then $x_{1}, y_{1}, x_{2}, y_{2}, \cdots$ is the desired basis of $\mathfrak{B}$.

Let $z_{1}, \cdots, z_{n}$ be an orthonormal basis of $\mathfrak{B}$ and let the matrix of $\sigma$ with respect to this basis be $\left(\alpha_{i j}\right)$. If $i \neq j$ exist with $\alpha_{i \imath}=\alpha_{j j}=0$, then $\mathfrak{U}=\left\langle z_{i}, z_{j}\right\rangle$ is the desired subspace. If no such $i, j$ exist then in any event there must exist $i, j$ with $\alpha_{i i} \neq \alpha_{j j}$ (otherwise we would have $0=\operatorname{tr}(\sigma)=n \alpha_{i i}$ and since $n \neq 0$, all $\left.\alpha_{\imath i}=0\right)$. Say $\alpha_{11} \neq \alpha_{22}$; then $\left\langle z_{1}, z_{2}, z_{3}\right\rangle=\mathfrak{W}$ is a nonisotropic subspace such that $\sigma_{\mathscr{2}}$ is not a scalar. By Lemma 4 applied to $\sigma_{\mathfrak{g}}$, there is a 2-dimensional subspace $\mathfrak{U}$ of $\mathfrak{B}$ such that $\sigma_{\mathfrak{u}}=\left(\sigma_{\mathfrak{2}}\right)_{\mathfrak{u}}$ has trace zero. This completes the proof.

Finally, we let $\mathfrak{B}$ be as in Lemma 5 and $\mathfrak{\Im}$ be the Jordan algebra of symmetric operators on $\mathfrak{B}$, and prove Theorem 2 for $\mathfrak{\Im}$. Let $\tau$ be a linear transformation on $\mathfrak{B}$ skew-symmetric with respect to the form. Then $\tau=\left[\sigma_{1}, \sigma_{2}\right]=\sigma_{1} \sigma_{2}-\sigma_{2} \sigma_{1}$ for some $\sigma_{1}, \sigma_{2} \in \mathfrak{J}$. Indeed if $z_{1}, \cdots, z_{n}$ is an orthonormal basis of $\mathfrak{B}$, then the matrix of $\tau$ with respect to this basis is skew-symmetric, while $\breve{\checkmark}$ consists of all transformations with symmetric matrices. I.e., $\tau=\sum_{\imath<j} \alpha_{i j}\left(e_{i j}-e_{j i}\right)$, where the $e_{i j}$ are the usual matrix units in $\operatorname{Hom}(\mathfrak{B}, \mathfrak{B})$. If $\beta_{1}, \cdots, \beta_{n} \in \Phi$ are distinct, then $\left[e_{i j}, \sum_{k} \beta_{k} e_{k k}\right]=\left(\beta_{j}-\beta_{i}\right) e_{i j}$ and so

$$
\left[\sum_{i<j} \frac{\alpha_{i j}}{\beta_{j}-\beta_{i}}\left(e_{i j}+e_{j}\right), \sum_{k} \beta_{k} e_{k k}\right]=\tau .
$$

Now let $\sigma \in \mathfrak{\Im}$ have trace zero and choose a hyperbolic basis $x_{1}, y_{1}$, $\cdots, x_{l}, y_{l}$, and (possibly) $z$ as in Lemma 5 . Let $\beta_{1}, \cdots, \beta_{l} \in \Phi$ be such that $\beta_{1}, \cdots, \beta_{l},-\beta_{1}, \cdots,-\beta_{l}, 0$ are all distinct. Let $\tau$ be the linear transformation whose matrix with respect to the hyperbolic basis is diagonal, with diagonal entries (in order) $\beta_{1},-\beta_{1}, \beta_{2},-\beta_{2}, \cdots, \beta_{l},-\beta_{l}$, and (possibly) 0. Then $\tau$ is skew-symmetric. We rename the diagonal entries of $\tau$, calling them $\gamma_{1}, \gamma_{2}, \cdots, \gamma_{2 l}$, and (possibly) $\gamma_{2 l+1}$. The eigenvalues of the map ad $\tau: \rho \rightarrow[\tau, \rho]$ for all $\rho \in \operatorname{Hom}(\mathfrak{B}, \mathfrak{B})$ are 0 , $\gamma_{i}-\gamma_{j}(i \neq j)$; the kernel of ad $\tau$ is the set of diagonal matrices (the $\gamma_{i}$ are distinct), and ad $\tau$ maps $\operatorname{Hom}(\mathfrak{B}, \mathfrak{B})$ into the space of matrices 
all of whose diagonal entries are 0 . Also ad $\tau$ stabilizes $\Im$ (since $\tau$ is skew), hence stabilizes $\mathfrak{\Im}_{1}=\{\rho \in \mathfrak{\Im} \mid$ the matrix of $\rho$ has all diagonal entries zero\}. Since ad $\tau \mid \mathfrak{\Im}_{1}$ is nonsingular, we conclude that ad $\tau$ maps $\mathfrak{\Im}_{1}$ onto $\mathfrak{\Im}_{1}$. I.e., $\sigma=[\tau, \rho]$ for some $\rho \in \mathfrak{\Im}_{1}$. Now the previous paragraph implies that $\tau=\left[\rho_{1}, \rho_{2}\right]$ for some $\rho_{1}, \rho_{2} \in \mathfrak{\Im}$. So

$$
\sigma=\left[\left[\rho_{1} \rho_{2}\right] \rho\right]=\frac{1}{4}\left(\left(\rho_{2} \cdot \rho\right) \cdot \rho_{1}-\rho_{2} \cdot\left(\rho \cdot \rho_{1}\right)\right) .
$$

Hence $\sigma$ is an associator in $\mathfrak{\Im}$. This proves Theorem 2.

We note that, by the computations in $[4, \S 5]$, Lemmas 3 and 5 may be stated in the following single conjugacy theorem.

THEOREM 3. Let $\mathfrak{\Im}$ be a simple Jordan algebra over an algebraically closed field $\Phi$ of characteristic equal to 0 or bigger than the degree of $\mathfrak{\Im}$. Let $\mathfrak{F}$ be a Cartan subalgebra of the derivation algebra of $\mathfrak{\Im}$ and let $\mathfrak{\Im}=\sum_{\alpha} \mathfrak{\Im}_{\alpha}$ be the corresponding decomposition of $\mathfrak{\Im}$ into weight spaces relative to $\mathfrak{F}$. Then any element of $\mathfrak{\Im}$ of trace 0 is conjugate under the automorphism group of $\mathfrak{\Im}$ to an element of $\sum_{\alpha \neq 0} \mathfrak{\Im}_{\alpha}$, the sum of the nonzero weight spaces.

It would be interesting to have a more conceptual, Lie-theoretic proof of Theorem 3.

We conclude with an open question: if $\mathfrak{\Im}$ is as in Theorem 3 , is it true that any derivation of $\mathfrak{\Im}$ has the form $\left[R_{x}, R_{y}\right]$ for some $x, y \in \mathfrak{F}$ (where $R_{x} \in \operatorname{Hom}(\Im, \mathfrak{\Im})$ is defined as usual by $R_{x}(\alpha)=x . \alpha$ )? We saw above that this holds if $\mathfrak{\Im}$ is the algebra of $n \times n$ symmetric matrices. Theorem 3 and this result (if true) together easily imply Theorem 2.

\section{REFERENCES}

1. A. A. Albert and B. Muckenhoupt, On matrices of trace zero, Michigan Math. J., 4 (1957), 1-3.

2. F. van der Blij and T. A. Springer, The arithmetics of octaves and of the group $G_{2}$, Neder. Akad. Wetensch. Proc. Ser. A62=Indag. Math., 21 (1959), 406-418.

3. G. Brown, On commutators in a simple Lie algebra, Proc. Amer. Math. Soc., 14 (1963), 763-767.

4. S. R. Gordon, The components of the automorphism group of a Jordan algebra, Trans. Amer. Math. Soc., 153 (1971), 1-52.

5. N. Jacobson, Structure and Representations of Jordan Algebras, Amer. Math. Soc. Colloq. Publ., Vol. 39, Providence, R. I., 1968.

6. R. D. Schafer, An Introduction to Nonassociative Algebras, Academic Press, New York, 1966.

7. K. Shoda, Einige Sätze über Matrizen, Japan J. Math., 13 (1937), 361-365.

Received November 29, 1972. This work was supported in part by NSF grant GP-23998.

University of California, Riverside 



\section{PACIFIC JOURNAL OF MATHEMATICS}

\section{EDITORS}

RICHARD ARENS (Managing Editor)

University of California

Los Angeles, California 90024

R. A. BeAumont

University of Washington

Seattle, Washington 98105
J. DugundJI*

Department of Mathematics

University of Southern California

Los Angeles, California 90007

D. Gilbarg and J. Milgram

Stanford University

Stanford, California 94305

\section{ASSOCIATE EDITORS}

E. F. BECKENBACH

B. H. NEUMANN

F. WOLF

K. YoSHIDA

\section{SUPPORTING INSTITUTIONS}

UNIVERSITY OF BRITISH COLUMBIA
CALIFORNIA INSTITUTE OF TECHNOLOGY
UNIVERSITY OF CALIFORNIA
MONTANA STATE UNIVERSITY
UNIVERSITY OF NEVADA
NEW MEXICO STATE UNIVERSITY
OREGON STATE UNIVERSITY
UNIVERSITY OF OREGON
OSAKA UNIVERSITY

UNIVERSITY OF BRITISH COLUMBIA CALIFORNIA INSTITUTE OF TECHNOLOGY UNIVERSITY OF CALIFORNIA MONTANA STATE UNIVERSITY NEW MEXICO STATE UNIVERSITY UNIVERSITY OF OREGON OSAKA UNIVERSITY
UNIVERSITY OF SOUTHERN CALIFORNIA STANFORD UNIVERSITY UNIVERSITY OF TOKYO UNIVERSITY OF UTAH WASHINGTON STATE UNIVERSITY UNIVERSITY OF WASHINGTON AMERICAN MATHEMATICAL SOCIETY NAVAL WEAPONS CENTER

* C. R. DePrima California Institute of Technology, Pasadena, CA 91109, will replace J. Dugundji until August 1974. 


\section{Pacific Journal of Mathematics}

\section{Vol. 51, No. $1 \quad$ November, 1974}

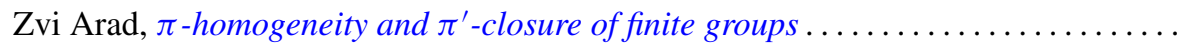

Ivan Baggs, A connected Hausdorff space which is not contained in a maximal

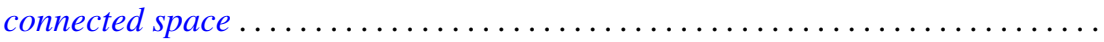

Eric Bedford, The Dirichlet problem for some overdetermined systems on the unit ball in $C^{n}$

R. H. Bing, Woodrow Wilson Bledsoe and R. Daniel Mauldin, Sets generated by

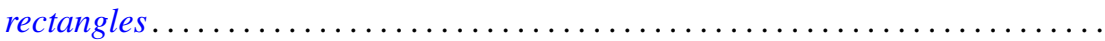

Carlo Cecchini and Alessandro Figà-Talamanca, Projections of uniqueness for

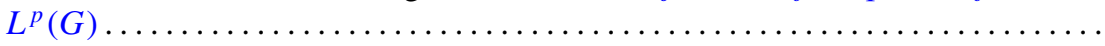

Gokulananda Das and Ram N. Mohapatra, The non absolute Nörlund summability of Fourier series .

Frank Rimi DeMeyer, On separable polynomials over a commutative ring ........ Richard Detmer, Sets which are tame in arcs in $E^{3} \ldots \ldots \ldots \ldots \ldots \ldots \ldots \ldots$

William Erb Dietrich, Ideals in convolution algebras on Abelian groups ..........

Bryce L. Elkins, A Galois theory for linear topological rings .................

William Alan Feldman, A characterization of the topology of compact convergence on $C(X)$.

Hillel Halkin Gershenson, A problem in compact Lie groups and framed cobordism

Samuel R. Gordon, Associators in simple algebras.

Marvin J. Greenberg, Strictly local solutions of Diophantine equations

Jon Craig Helton, Product integrals and inverses in normed rings . . . . . . . . . . . .

Domingo Antonio Herrero, Inner functions under uniform topology . . .

Jerry Alan Johnson, Lipschitz spaces .

Marvin Stanford Keener, Oscillatory solutions and multi-point boundary value

functions for certain nth-order linear ordinary differential equations.

John Cronan Kieffer, A simple proof of the Moy-Perez generalization of the

Shannon-McMillan theorem .......................

Joong Ho Kim, Power invariant rings

Gangaram S. Ladde and V. Lakshmikantham, On flow-invariant sets .

Roger T. Lewis, Oscillation and nonoscillation criteria for some self-adjoint even

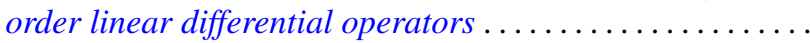

Jürg Thomas Marti, On the existence of support points of solid convex sets ..

John Rowlay Martin, Determining knot types from diagrams of knots . .

James Jerome Metzger, Local ideals in a topological algebra of entire functions

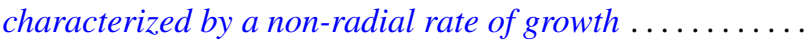

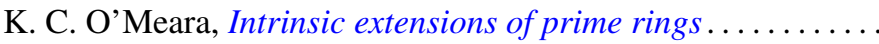

Stanley Poreda, A note on the continuity of best polynomial approximations ..

Robert John Sacker, Asymptotic approach to periodic orbits and local prolongations

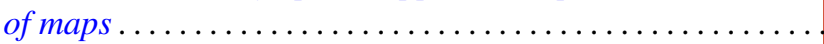

Eric Peter Smith, The Garabedian function of an arbitrary compact set . .

Arne Stray, Pointwise bounded approximation by functions satisfying a side condition

John St. Clair Werth, Jr., Maximal pure subgroups of torsion complete abelian

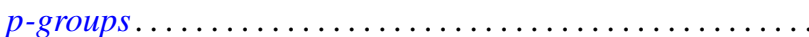

